\title{
Kinematics and Energetics of A Solar Jet from NOAA Active Region 12297
}

\author{
Reetika Joshi and Ramesh Chandra \\ Department of Physics, DSB Campus, Kumaun University, Nainital - 263 001, India \\ Email: reetikajoshi.ntl@gmail.com
}

\begin{abstract}
In this article, we have presented the study of a solar jet on March 14, 2015 recorded from the Atmospheric Imaging Assembly (AIA) onboard Solar Dynamics Observatory (SDO) satellite. The jet was observed from the solar active region NOAA AR 12297. We have discussed the dynamics and calculated the various possible energy contents of the observed jet. The estimated total energy flux liberated during the jet ejection was $2.1 \times 10^{7} \mathrm{erg} \mathrm{cm}^{-2} \mathrm{~s}^{-1}$.
\end{abstract}

Keywords: Solar Jets, SDO, energy flux, Solar activities.

\section{Introduction}

Based on size and time scales, various types of activities are observed on the solar surface. These activities can be divided into two categories namely small and large scale activities. The small scale activities are solar jets/surges, micro flares, etc whereas, the large solar activities includes solar filament eruptions, solar flares, Coronal Mass Ejections (CMEs) etc. Together with the large scale activities, which directly or indirectly affect our space weather, the small scale solar activities are also very important and crucial. The reason is that their physical mechanism is similar to large scale solar events. Among them one of the important solar activity is solar jets. Because of their small source region and high speeds, they are termed as jets. According to various observational studies, their heights, speeds, and widths are $10^{4}-4 \times 10^{5} \mathrm{~km}, 100-800 \mathrm{~km} \mathrm{~s}^{-1}$, and $2 \times 10^{3}$ $10^{5} \mathrm{~km}$ respectively (Shimojo et al., 1996; Nistico et al., 2009; Chandra et al., 2017a, Joshi et al., 2017). It is now commonly accepted that magnetic reconnection the mechanism for the energy release in solar jets (Yokoyama and Shibata, 1995, Torok, et al., CSHARAD
2009, Zuccarello et al., 2017). Based on this mechanism several numerical simulation have been done. However, we still don't know how this magnetic reconnection is initiated for the triggering of solar jets. We have reported in this paper, the kinematics and energetics of a solar jet on March 14, 2015. The paper is organized as follows; in section 2 we describe the observations and results. We discussed and summarized our findings in section 3.

\section{Observations and Results}

For this current study, We have used the data from the AIA (Lemen et al., 2012) onboard SDO (Pesnell, Thompson, and Chamberlin, 2012). The spatial and temporal resolution of AIA data are $1.2^{\prime \prime}$ and $12 \mathrm{~s}$ respectively. The morphology and the energetics derived for this jet are discussed in following subsections;

\subsection{Morphology}

We have observed the jet on March 14, 2015 from NOAA active region 12297 towards the south-west direction (S17W26). The jet http://jmr.sharadpauri.org 
J. Mountain Res. ISSN: 0974-3030

DOI: https://doi.org/10.51220/jmr.v14il.10 Vol. 14 (1), (2019) 43-46 
started at 11:43 UT and reached its peak at 11:56 UT. The evolution of the jet in AIA 304 $\AA$ is shown in Figure 1. The jet was associated with a filament eruption on March 15, 2015, which has been studied by Chandra et al., 2017b.
2.2 Kinematics and Energetics To probe the kinematics, we have done a time-distance analysis of the jet in AIA $304 \AA$, which is shown in Figure 2.

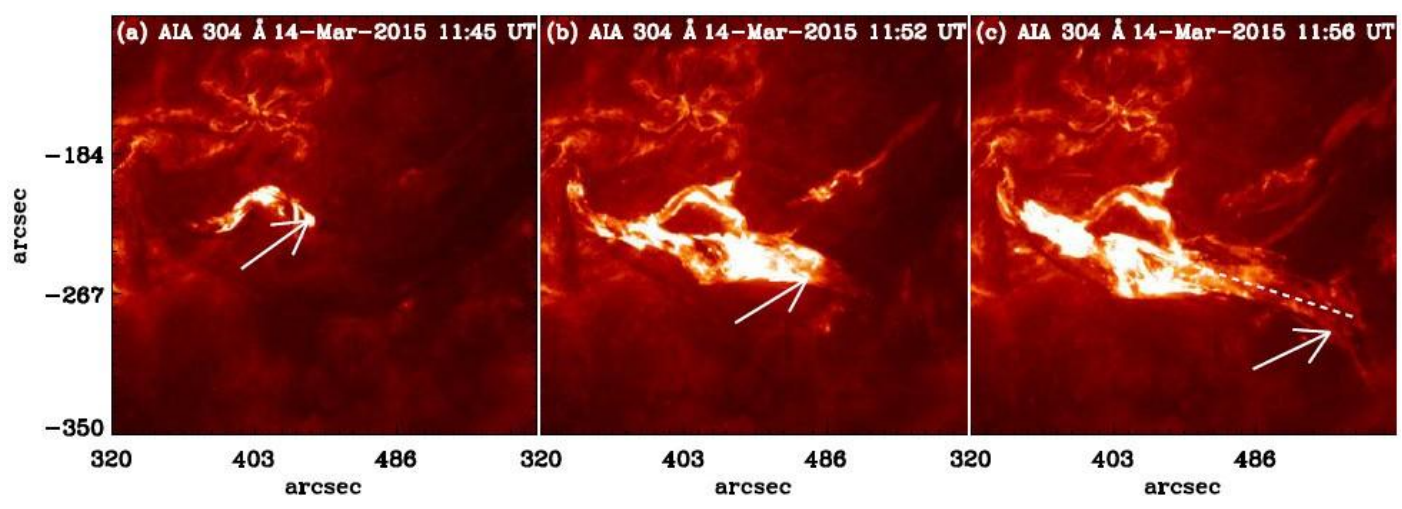

Figure 1 (Color online) : Spatial evolution of jet observed in AIA $304 \AA$, shown by white arrows. The white dashed line shows the position of the slit along which the time-distance analysis is drawn.

In this method, we fixed a slit at the centre of the jet and then observed the motion of plasma along that slit. We have calculated the velocity and the maximum extension of the jet, from this analysis, and we found the values as $180 \mathrm{~km} \mathrm{~s}^{-1}$ and $85 \mathrm{Mm}$. For the density distribution of plasma material with a given temperature range, we performed the emission measure (EM) analysis developed by Hannah \& Kontar 2012, with six different AIA channels (94 ̊̊, $131 \AA$, $171 \AA, 193 \AA$,

$211 \AA$, and $335 \AA$ ) and shown as Figure 3. We computed the different energy components that contribute to the total energy flux of the triggered jet following the equation shown in equation 1 proposed by Pucci et al., 2013 .

$\mathrm{F}_{\text {Total }}=\mathrm{F}_{\mathrm{K}}+\mathrm{F}_{\mathrm{P}}+\mathrm{F}_{\mathrm{W}}+\mathrm{F}_{\mathrm{R}}+\mathrm{F}_{\mathrm{E}}(1)$

Where, $F_{K}, F_{P}, F_{W}, F_{R}$, and $F_{E}$ are the kinetic energy flux, potential energy flux, wave energy flux, radiativ energy flux, and enthalpy energy flux respectively. These different components of energy fluxes are defined as:

$$
\begin{aligned}
& \mathrm{F}_{\mathrm{K}}={ }_{2}^{1} \rho v^{\mathrm{g}} \\
& \mathrm{F}_{\mathrm{P}}=\rho g g^{l} \\
& \mathrm{~F}_{\mathrm{W}}=\sqrt{\frac{\infty}{4 \pi}} \xi^{2} \mathrm{~B} \\
& \mathrm{~F}_{\mathrm{R}}=n_{\mathrm{E}}^{2} \chi^{T^{\alpha} l} \\
& \mathrm{~F}_{\mathrm{E}}=\underset{y-1}{y} p w
\end{aligned}
$$

where $\rho, w_{0}, \mathbb{B}, B$ and $\mathrm{g}$ represents the mass density of plasma material, outflow speed of the ejected plasma, solar gravitational acceleration, maximum vertical extension of the jet, magnetic field strength, 


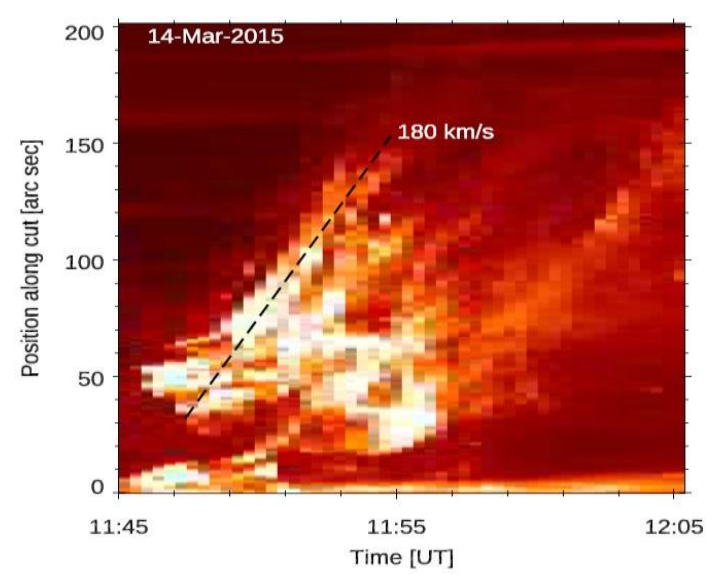

Figure 2 (Color online): Time-Distance image of the jet along the slit shown in figure 1 (c). electron density, and temperature of the jet. $\xi$ is the amplitude of non-thermal motion in the jet, here we are taking $\xi=100 \mathrm{~km} \mathrm{~s}^{-1}$ from the results of Kim et al., 2007 and $y={ }_{3}^{5}, a=0, x=10^{-34.94}$ with $x^{T^{1 \times}}$ in W $\mathrm{m}^{3}$ and $\mathrm{B}=2.8 \mathrm{G}$ from Pucci et al., 2013. From Figure 3, we have noted that the temperature and the total emission measure $(\mathrm{EM})$ is $\sim 1.6 \mathrm{MK}$ and $5 \times 10^{26} \mathrm{~cm}^{-5}$.

Followed these values we get the electron number density for the plasma material,

$n_{g}=\sqrt{m}$

which comes out to be $7.9 \times 10^{8} \mathrm{~cm}^{-3}$, here w is the width of the jet, which is $\sim 8 \mathrm{Mm}$ in this case. By using these various known values, we evaluated the different energy contributions from equations (2) to (6) as curves at the footpoint of the jet (black line) and pre jet (red line) phase.

$\mathrm{F}_{\mathrm{K}}=4.0 \times 10^{6} \mathrm{erg} \mathrm{cm}^{-2} \mathrm{~s}^{-1}, \mathrm{~F}_{\mathrm{P}}=5.6 \times 10^{6} \mathrm{erg}$ $\mathrm{cm}^{-2} \mathrm{~s}^{-1}, \mathrm{~F}_{\mathrm{W}}=2.9 \times 10^{6} \mathrm{erg} \mathrm{cm}^{-2} \mathrm{~s}^{-1}, \mathrm{~F}_{\mathrm{R}}=0.53$ $\mathrm{x} 10^{6} \mathrm{erg} \mathrm{cm}^{-2} \mathrm{~s}^{-1}$, and $\mathrm{F}_{\mathrm{E}}=8.1 \times 10^{6} \mathrm{erg} \mathrm{cm}^{-2} \mathrm{~s}^{-}$ 1

OSHARAD 45
From equation (1) the total energy flux liberated with the jet ejection comes out to be $\mathrm{F}_{\text {total }}=2.1 \times 10^{7} \mathrm{erg} \mathrm{cm}^{-2} \mathrm{~s}^{-1}$ These values are in agreement with the previous observations by Pucci et al., 2013 and Paraschiv et al., 2015.
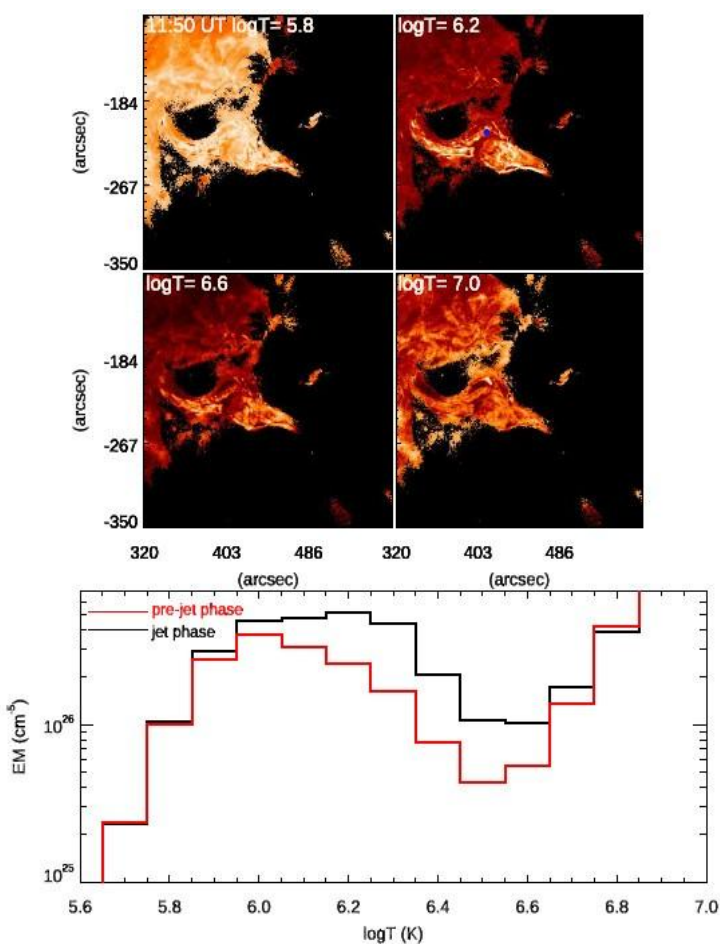

Figure 3 (Color online): Top panel: This shows the different EM maps of the jet at different temperatures. The blue circle in the top panel shows the location which have use for the EM analysis. Bottom panel: EM

\section{Summary}

The jet observed by SDO/AIA on March 14, 2015 was selected and studied for various physical parameters. In this paper, we have presented the kinematics and the energetics of the jet associated with a filament eruption on March 15, 2015. The Summary of this study is as follows:

- The jet was ejected with a speed of 180 $\mathrm{km} \mathrm{s}^{-1}$ and height $85 \mathrm{Mm}$. 
- The total energy flux contributed from the different energy components of the jet was $2.1 \times 10^{7} \mathrm{erg} \mathrm{cm}^{-2} \mathrm{~s}^{-1}$.

- We have found that the maximum contribution in the total energy flux is from the enthalpy energy and the minimum contribution is from the radiative energy. This energy flux is useful to calculate the energy rate supplied to the corona, during the jet ejection which will give an estimate of jet's contribution on coronal heating.

\section{Acknowledgements}

The authors are thankful to the open data policy of SDO/AIA. RJ was supported by DST, New Delhi, India as an INSPIRE fellow.

\section{References}

Chandra, R., et al. 2017a, A\&A, 598, A41. Chandra, R., et al., Sol. Phys., 2017b, 292, 81.

Joshi, R., et al. 2017, Sol. Phys., 292, 152. Hannah, I. G., \& Kontar, E. P. 2012, A\&A, 539, A146

Lemen, J.R., et al., 2012, Sol. Phys., 275, 17.

Nisticò, G., et al., 2009, Sol. Phys., 259, 87. Paraschiv, A. R., Bemporad, A., Sterling, A.C., 2015, A\&A, 579, A96.

Pesnell, W.D., Thompson, B.J., Chamberlin, P.C., 2012, Sol. Phys., 275, 3.

Pucci, S., et al., 2013, ApJ, 776, 16.

Shimojo, M., et al. 1996, PASJ, 48, 123.

Török, T., et al., 2009, ApJ, 704, 485.

Yokoyama, T., Shibata, K.: 1995, Nature 375, 42

Zuccarello, F. P, et al.,, A \& A, 2017, 601, A26

$* * * * * * * *$ 